\title{
Novel Electropolishing of Pure Metallic Titanium in Choline Chloride-Based Various Organic Solvents
}

\author{
Wrya. O. KARIM, ${ }^{a, *}$ Jamil. A. JUMA, ${ }^{b}$ Khalid M. OMER, ${ }^{a}$ Nawzad N. AHMAD, ${ }^{a}$ \\ Dana A. KADER, ${ }^{a}$ Brwa B. TOFIQ, ${ }^{c}$ and Shujahadeen B. AZIZ ${ }^{\mathrm{c}}$ \\ a Chemistry Department, College of Science, University of Sulaimani, Qliasan Street, Slemani City, 46002, Kurdistan, Iraq
b Department of Chemistry, Faculty of Science and Health, Koya University, KOY45, Kurdistan Region-Iraq
'Advanced Polymeric Materials Research Lab., Department of Physics, College of Science, University of Sulaimani,
Qlyasan Street, Sulaimani 46002, Kurdistan Region-Iraq
}

*Corresponding author: wrya.karim@univsul.edu.iq

\begin{abstract}
In this report we present a straightforward and efficient electropolishing methodology of pure metallic titanium in two different electrolytic baths; choline chloride-ethanol ( $\mathrm{ChCl}$-eth.) and choline chloride-methanol-butanol (ChCl-meth.-but.) in molar ratios of $1: 4$ and $1: 6: 2$, respectively at $20^{\circ} \mathrm{C}$. The electrolytic baths under study possess relatively low-cost and environmentally benign. In the electropolishing processes, potentiostatic technique was applied. For the surface characterizations both atomic force microscope (AFM) and scanning electron microscopy (SEM) were used. The results showed that titanium surface was polished electrochemically at nanoscale and relatively free from defects. The working voltages of 2 and $1.2 \mathrm{~V}$ were applied in $\mathrm{ChCl}$-eth. and $\mathrm{ChCl}$-meth.-but., respectively within a time scale of 40 minutes in the present electrolytic baths. The roughness of pure metallic titanium (ca. $120.5 \mathrm{~nm}$ ) was reduced to $0.10 \mathrm{~nm}$ in ChCl-eth. and $0.66 \mathrm{~nm}$ in $\mathrm{ChCl}$-meth.-but.
\end{abstract}

(c) The Author(s) 2020. Published by ECSJ. This is an open access article distributed under the terms of the Creative Commons Attribution 4.0 License (CC BY http://creativecommons.org/licenses/by/4.0/), which permits unrestricted reuse of the work in any medium provided the original work is properly cited. [DOI: 10.5796/electrochemistry.20-00120].

Keywords : Deep Eutectic Solvent, Ionic Liquid, Titanium, Electropolishing

\section{Introduction}

Electropolishing protocol is the process of reducing surface roughness of metals and alloys using optimised anodic dissolution in an appropriate electrolytic bath. ${ }^{1}$ Pure metallic titanium and its alloys have been focused as invaluable materials in medicine field. ${ }^{2,3}$ To reduce the rate of corrosion of metallic materials, electropolishing has been carried out. ${ }^{4}$ The mechanism of electropolishing in aqueous media has comprehensively been understood; whereas that in ionic liquids and deep eutectic solvents is still in early stage. ${ }^{1,5}$ Four decisive parameters have been taken into consideration to carry out a successful electropolishing process; electrolyte bath composition, temperature, voltage and current density. ${ }^{1,5}$

Throughout the literature, the most focused metallic materials in the electropolishing are stainless steels in aqueous and ionic liquids media. ${ }^{6-9}$ It is also a few work on titanium, nickel and cobalt surface finishing were documented using ionic liquids. ${ }^{3,10}$ Abbott et al. is one of the pioneers that has own perspective regarding usage of deep eutectic solvents. ${ }^{11,12}$

The majority of electropolishing of titanium were conducted in aqueous media based on perchloric acid, such as perchloric acid/ acetic acid and perchloric acid/methanol/ethylene glycol and perchloric acid-free electrolyte, for instance methanol/sulfuric acid. ${ }^{13-15}$ Recently, sodium chloride/ethylene glycol was used in electropolishing titanium. ${ }^{16}$

As attractive electrolytic bath media, ionic liquids (ILs) and deep eutectic solvents (DESs) have drawn attraction of a number of research groups, particularly in anodic dissolution and electroplating. Ionic liquids are liquid mixtures of discrete ions of relatively large organic cations and anion, for example, 1-butyl-3-methylimidazolium chloride $\left[\mathrm{C}_{4} \mathrm{~min}\right][\mathrm{Cl}]$ below $100^{\circ} \mathrm{C} .{ }^{17,18}$ Deep eutectic solvents (DESs) are eutectic liquid mixtures of complex systems of variety of ions, for instance choline chloride and ethylene glycol. ${ }^{19,20}$
In the present study, the impact of electrolyte composition on electropolishing of pure metallic titanium as one of invaluable metals is addressed. It is also essential to answer an importance of usage of environmentally benign electrolytic bath in the electrochemical polishing. It will be clarified that the electrolyte composition has substantial impact on the successfulness of the process as a way of metallic surface enhancement against corrosion.

\section{Experimental}

\subsection{Materials and methods}

The Ti sheet was purchased from Alfa Aesar (Alfa Aesar 99\%, USA). The titanium surface was cleaned using $0.01 \mathrm{M}$ of dilute sulfuric acid and $0.01 \mathrm{M}$ of hydrochloric acid mixture prior to electropolishing process. Afterwards, it was further cleaned by insertion into acetone with sonication to degreasing. The choline chloride ( $\mathrm{ChCl}$, 99\%, Sigma Aldrich), methanol (meth., 99.8\%, Sigma Aldrich, USA), ethanol (eth. $\geq 99.8 \%$, Sigma Aldrich, USA), n-butanol (But., 99.8\%, Sigma Aldrich, USA) were purchased from Sigma Aldrich and used as received.

For plotting the current density-voltage $(j-V)$ and current density-time $(j-t)$, AC/DC Laboratory Power Supply DC: 0-30 V and digital multi-meters (Widewing AN8008 True-RMS) were used. For examining the morphology, scanning electron microscopy (Philips XL30 ESEM) was used. The atomic force microscope (AFM) was used ex situ using a Digital Instruments Nanoscope 4 run at contact mode operation. The acquisition of images was conducted in resonant mode at a frequency $(300 \mathrm{~Hz})$ at a scan rate of $0.5 \mathrm{~Hz}$.

The first electrolytic bath (ChCl-Meth.-but.) comprises mixing choline chloride, methanol and butanol in a $1: 6: 2$ mole ratio. The mixture was shaken vigorously until a clear and homogeneous liquid was gained. 

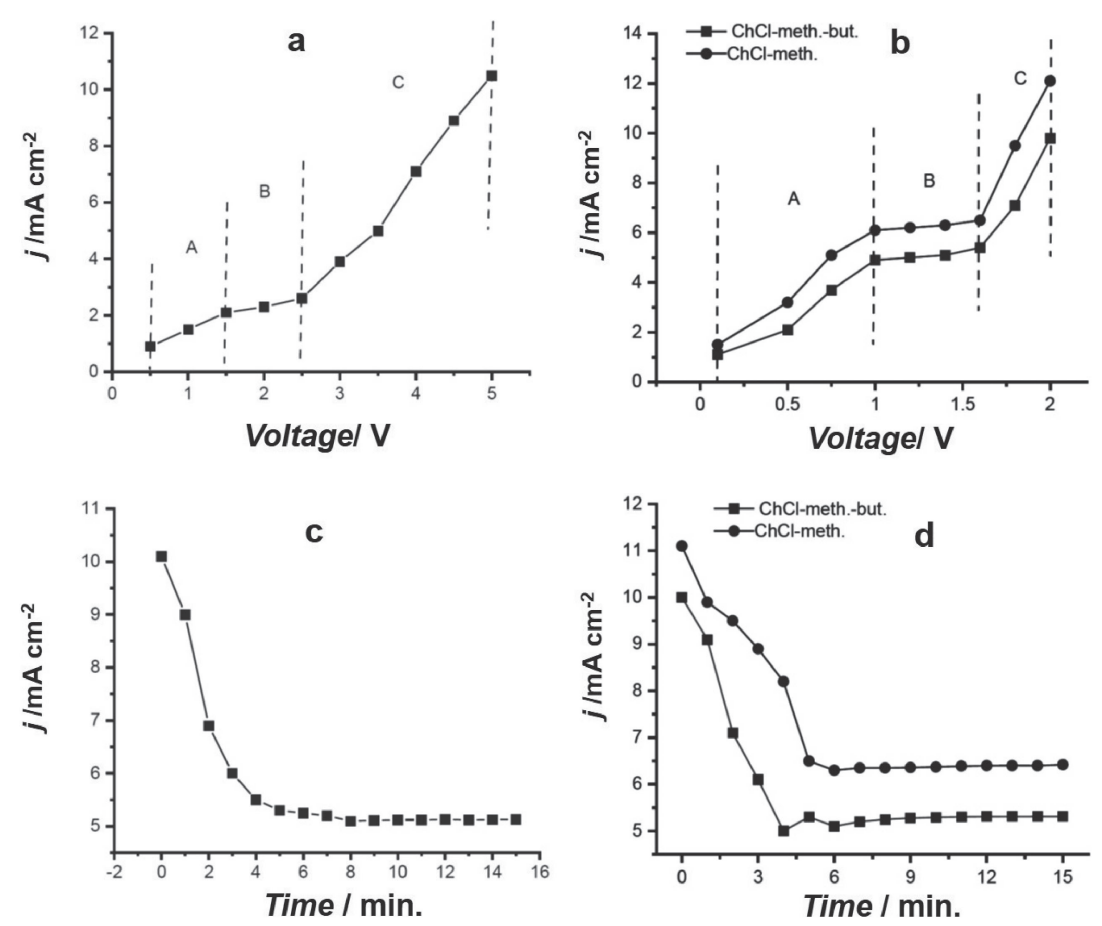

Figure 1. Potentiostatic profiles of pure metallic titanium in (a) ChCl-eth. and (b) ChCl-meth.-but. and ChCl-meth., and $j$ - $t$ transient curves of pure metallic titanium in (c) ChCl-eth. at $2 \mathrm{~V}$ and (d) ChCl-meth.-but., and ChCl-meth. at $1.2 \mathrm{~V}$. All electrochemical measurements at $20{ }^{\circ} \mathrm{C}$.

The second electrolytic bath (ChCl-eth.) preparation involves mixing choline chloride and ethanol in a molar ratio of $1: 4$. The clear and homogeneous liquid was obtained after shaking of the two chemicals.

The system of two electrodes was used in the electropolishing process. The measurements were performed using $1 \mathrm{~cm}^{2}$ Ti disk (thickness of $2 \mathrm{~mm}$ ) and cylindrical platinum metal as working and counter electrodes, respectively in $10 \mathrm{ml}$ of the electrolytic baths. The electropolishing results of the working voltage at 2 and $1.2 \mathrm{~V}$ for 40 minutes at $20^{\circ} \mathrm{C}$ were presented. During electrochemical experiments, the distance of $1 \mathrm{~cm}$ was maintained between the working and the counter electrodes.

\section{Results and Discussion}

\subsection{Electrochemical measurements}

The $j-V$ profiles of pure metallic titanium electrode in the $\mathrm{ChCl}$ eth., and ChCl-meth.-but. mixtures at $20^{\circ} \mathrm{C}$ is shown in Figs. 1a and 1b. It is seen that in both electrolytic baths, the responses are quite comparable with slight differences in current values. Three distinct ranges are the feature of both responses where denoted as A, B and $\mathrm{C}$ that represent gradual current ascending, plateau current and steeply current rise, respectively. ${ }^{14,21}$ In range A, there is a metallic titanium dissolution randomly followed by effective electropolishing in range $\mathrm{B}$ and electrolytic decomposition occurs at range $\mathrm{C}$. In range $\mathrm{A}$, there is a pure metallic dissolution electrochemically starting from 0.5 and $0.1 \mathrm{~V}$ in $\mathrm{ChCl}$-eth. and in ChCl-meth.-but., respectively. This might be due to facilitation of mass transport through the interfacial region in the later. Regarding range $\mathrm{B}$, the onset voltages are $1.5 \mathrm{~V}$ and $1 \mathrm{~V}$ in ChCl-eth. and ChCl-meth.-but., respectively. The low onset voltage in $\mathrm{ChCl}$-meth.-but. is ascribed to the kinetic factor which is favorable. In an electropolishing process of titanium using a mixture of perchloric acid-methanol-ethylene glycol, a relatively wide voltage range were recorded by Nasirpouri and Peighambardoust. ${ }^{14}$ The superiority of the present electrolytic baths is that the electropolishing of titanium can successfully be performed at much lower voltage by ten times. Herein, the effective electropolishing ranges are $1.5-2.5 \mathrm{~V}$ and $1-1.6 \mathrm{~V}$ correspond to $\mathrm{ChCl}$-eth., and $\mathrm{ChCl}$-meth.-but. mixtures, respectively. The ranges are relatively lower and narrower in both electrolytic baths under study than previously reported in a comparable medium. ${ }^{21}$

The closeness of electrochemical responses in ChCl-meth.-but. and ChCl-meth. baths are correlated to electrolytic bath compositions except the existence of butanol in the former one as shown in Fig. 1b. However, the presence of butanol causes recording low current values over the electrochemical time scale. This can be attributed to the lower chloride concentration in case of presence of butanol in the electrolytic bath. Moreover, the high possibility of complexation of titanium in the electrolytic baths under study is with chloride ion. Figures $1 \mathrm{c}$ and $1 \mathrm{~d}$ show the $j-t$ transient responses of titanium in both electrolytic baths where obvious steady states are seen. It is also noticed that electropolishing process commences after 5 minutes of the experimental time scale. It is evidenced that during the steady state, mass transport governs the metallic titanium dissolution in the interfacial region other than electrochemical migration.

In the electropolishing process, pure metallic titanium is presumably dissolved electrochemically in the form of titanium/ chloride $\left[\mathrm{TiCl}_{4}\right]$ as shown below: ${ }^{1}$

$$
\mathrm{Ti}+4 \mathrm{Cl}^{-} \rightarrow \mathrm{TiCl}_{4(\mathrm{l})}+4 \mathrm{e}^{-}
$$

The relatively low working voltages (ca. 1.2 and $2 \mathrm{~V}$ ) in the current work compared to the high voltage (ca. $12 \mathrm{~V})^{21}$ in the previous study using choline chloride/ethylene glycol is due to the more viscous film formation in the later one. The higher viscosity of the choline chloride-ethylene glycol than present electrolytic baths is correlated to the existence of a stronger network of hydrogen bonding in the former one.

\subsection{Surface morphology}

Electropolishing of pure metallic titanium was carried out in two electrolytic baths at $20^{\circ} \mathrm{C}$. The qualitative and quantitative analyses were performed using atomic force microscopy (AFM) ex situ and scanning electron microscope (SEM). 

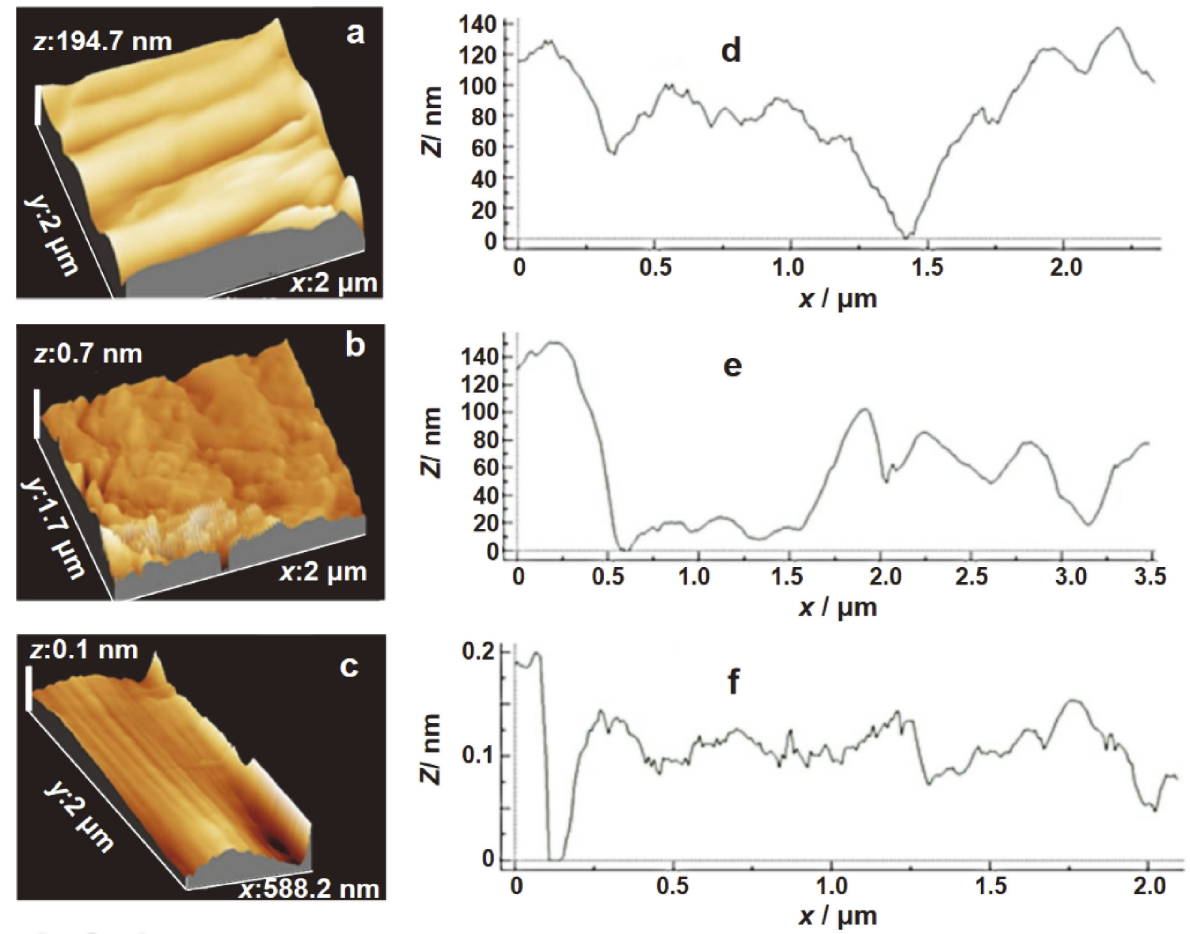

Figure 2. AFM images and single line traces alongside of pure metallic of $\mathrm{Ti}$ and polished samples recorded in resonant mode at a frequency (ca. $300 \mathrm{kHz}$ ) recorded in air at a scan rate of $0.5 \mathrm{~Hz}, 256$ lines: (a) AFM image of bare titanium, (b) AFM image of titanium electropolished in ChCl-meth.-but., (c) AFM image of titanium electropolished in ChCl-eth., (d) single line trace in AFM measurement of bare titanium, (e) single line trace in AFM measurement of electropolished titanium in ChCl-meth.-but., and (f) single line trace in AFM measurement of electropolished titanium in ChCl-eth.

\subsubsection{Atomic force microscopy (AFM)}

Figure 2 shows the AFM images of the pure metallic titanium before and after electropolishing in both electrolytic baths. Prior to electropolishing, over a length scale of $2 \times 2 \mu \mathrm{m}$, the titanium surface is characterized by many micro-roughness displaying sharp features in the $z$ direction across the whole sample as presented in Fig. 2a. It is seen that pure metallic titanium is relatively rough with feature sizes up to $Z_{\max }=194 \pm 5 \mathrm{~nm}$ (over 10 measurements) as shown in single line trace within $X=2.5 \mu \mathrm{m}$ in Fig. 2 d.

In ChCl-meth.-but. electrolyte, the electropolished surface over $2 \mu \mathrm{m} \times 1.7 \mu \mathrm{m}$ is presented in Fig. $2 \mathrm{~b}$. It is clearly seen that the polished area exhibits $Z_{\max }=0.70 \pm 0.01 \mathrm{~nm}$ (over 10 measurements). The electropolished surface of titanium in ChCl-eth. electrolyte is exhibited in Fig. 2c. Over $0.588 \mu \mathrm{m} \times 2 \mu \mathrm{m}$, the recorded $Z_{\max }=0.10 \pm 0.01 \mathrm{~nm}$ (over 10 measurements). Despite of relatively smooth surface of the polished areas, there is a variation in height to some extent across the scanned area as shown in Figs. 2e and 2f. In Fig. 2e, it is seen that there is an overlapping region between polished and unpolished around 0.5$1.5 \mu \mathrm{m}$ on the $x$-axis. The average roughness value obtained for electropolished pure metallic titanium in present work is much lower than that previously recorded $(2.53 \mathrm{~nm})$, especially in $\mathrm{ChCl}-$ eth. electrolyte. ${ }^{10}$

This can be ascribed to the presence of methanol and ethanol that provide a proper viscos layer over metallic titanium electrode that facilitates mass transport through it. In earlier study, the electropolished titanium surface possesses an average roughness of $5.7 \mathrm{~nm}$ which is higher than that of current results by almost 10 times. ${ }^{21}$ To explain this difference in electropolishing of pure metallic titanium, there is a common component in both electrolytes which is choline chloride and the difference is the presence of different organic solvents. It might be owing to optimum mass transport in present electrolytic baths.

\subsubsection{Scanning electron microscope (SEM)}

The unpolished and polished pure metallic titanium in the electrolytic baths at the boundary between the polished and unpolished regions are exhibited in Figs. $3 \mathrm{a}$ and $3 \mathrm{~b}$. From the SEM image in Fig. 3a, it is seen that the unpolished region possesses many grain boundaries whereas the polished one in $\mathrm{ChCl}$ meth.-but. is bright with no trace of features. It is worth-deserving that below $1 \mathrm{~V}$, a dull surface finished was obtained and above $1.6 \mathrm{~V}$, the electrolytic decomposition occurred. ${ }^{22}$ Regarding the electropolishing process of the metal in ChCl-eth., it is clearly seen that the pure metallic titanium is polished electrochemically at the optimum voltage of $2 \mathrm{~V}$ as shown in Fig. 3b. However, below $1.5 \mathrm{~V}$ and above $2.5 \mathrm{~V}$, pitting over the pure metallic titanium surface and electrolytic decomposition took place, respectively.

\section{Conclusions}

In conclusion, several decisive factors and superiority of titanium electropolishing in choline chloride-based methanol-butanol and ethanol electrolytic baths have been summarised as follows:

- The relative cheapness and benign nature of the presence electrolytic baths encourage researcher to perform electropolishing of pure metallic titanium in choline chloride-based organic solvents successfully.

- The presence of butanol is of great importance to avoid gas evolution at the anode, such as chlorine and oxygen. In other words, the butanol acts an electrolytic stabiliser against electrochemical perturbation.

- Both ChCl-meth.-but. and ChCl-eth. electrolytive baths are considered as promising media for achieving mirror-like surface of pure metallic titanium at $20^{\circ} \mathrm{C}$. This confirms the importance of electrolyte composition in determining the efficiency of electropolishing process of titanium. The $\mathrm{ChCl}-$ 


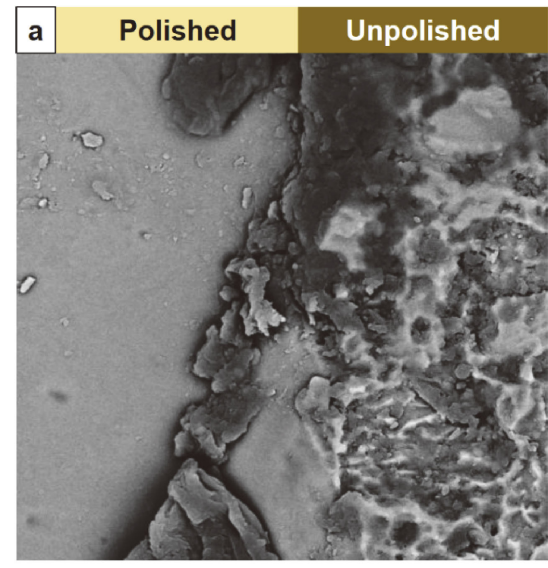

$10 \mu \mathrm{m}$

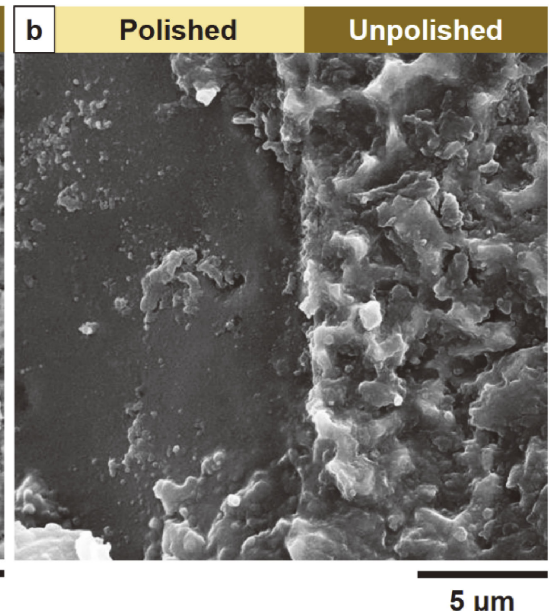

$5 \mu \mathrm{m}$

Figure 3. SEM images at the boundary between a polished and unpolished region of pure metallic titanium in (a) ChCl-meth.-but., at $1.2 \mathrm{~V}$ (magnification: $\times 5000$ ) and (b) ChCl-eth. at $2 \mathrm{~V}$ (magnification $\times 10000$ ) under working distance of $11.9 \mathrm{~mm}$ and acceleration voltage of $20 \mathrm{kV}$. (Both for 40 minutes at $20^{\circ} \mathrm{C}$ ).

eth. electrolytic bath provides slightly smoother surface than ChCl-meth.-but. electrolytic bath., despite of applying higher voltage.

- It is believed that the viscos layer over exposed titanium surface to the electrolytic baths is one of critical factor to achieve effective electropolishing by facilitating mass transport, in particular through interfacial region.

- The working voltages of 1.2 and $2 \mathrm{~V}$ in both media are relatively very low, providing less consuming electric energy during sufficient experimental time scale.

- Morphological study confirms smoothness in a wide scale (micrometer) by direct observation and the effectiveness of the electropolishing of pure metallic titanium in the current electrolytic baths.

\section{Acknowledgments}

The author would like to thank the University of Sulimani for funding.

\section{References}

1. W. Han and F. Fang, Int. J. Mach. Tools Manuf., 139, 1 (2019).

2. P. Barnes, A. Savva, K. Dixon, H. Bull, L. Rill, D. Karsann, S. Croft, J. Schimpf, and H. Xiong, Surf. Coat. Tech., 347, 150 (2018).

3. O. Lebedeva, G. Jungurova, D. Kultin, L. Kustov, A. Zankharov, K. Kalmikov, E. Chernikova, and V. Krasovskiy, Green Chem., 13, 1004 (2011).

4. P. Lopez-Ruiz, B. Garcia-Blanco, G. Vara, I. Fernández-Pariente, M. Guagliano, and S. Bagherifard, Appl. Surf. Sci., 492, 1 (2019).

5. S. Mohan, D. Kanagaraj, R. Sindhuja, S. Vijayalakshmi, and N. G. Renganathan, Trans. Inst. Met. Finish., 79, 140 (2001).

6. W. Han and F. Fang, J. Mater. Proc. Tech., 279, 116558 (2020).

7. P. Tyagi, T. Goulet, C. Riso, R. Stephenson, N. Chuenprateep, J. Schlitzer, C. Benton, and F. Garcia-Moreno, Addit. Manuf., 25, 32 (2019).

8. A. P. Abbott, G. Capper, K. J. Mckenzie, A. Glidle, and K. S. Ryder, Phys. Chem. Chem. Phys., 8, 4214 (2006).

9. A. P. Abbott, G. Capper, B. G. Swain, and D. A. Wheeler, Trans. Inst. Met. Finish., 83, 51 (2005).

10. W. O. Karim, A. P. Abbott, S. Cihangir, and K. S. Ryder, Trans. Inst. Met. Finish., 96, 200 (2018).

11. A. P. Abbott, G. Frisch, and K. S. Ryder, Annu. Rev. Mater, 43, 335 (2013).

12. A. P. Abbott, G. Frisch, J. Hartley, W. O. Karim, and K. S. Ryder, Prog. Nat. Sci. Mat. Int., 25, 595 (2015).

13. J. B. Mathieu and D. Landolt, J. Electrochem. Soc., 125, 1044 (1978)

14. N. S. Peighambardoust and F. Nasirpouri, Trans. Inst. Met. Finish., 92, 132 (2014).

15. O. Piotrowski, C. Madore, and D. Landolt, J. Electrochem. Soc., 145, 2362 (1998).

16. D. Kim, K. Son, D. Sung, Y. Kim, and W. Chung, Corros. Sci., 98, 494 (2015).

17. A. P. Abbott, G. Frisch, J. Hartley, W. O. Karim, and K. S. Ryder, Prog. Nat. Sci. Mat. Int., 25, 595 (2015).

18. W. O. Karim, S. B. Aziz, M. A. Brza, R. M. Abdullah, and M. F. Z. Kadir, App. Sci., 9, 4401 (2019).

19. T. A. Green, P. Vaverde, and S. Roy, J. Electrochem. Soc., 165, D313 (2018).

20. A. P. Abbott, K. J. McKenzi, and K. S. Ryder, "Technical Aspects" in Electrodeposition of Metals from Ionic Liquids (Eds. F. Endres and A. P. Abbott), Wiley VCH (2007).

21. W. O. Karim, J. A. Juma, K. M. Omer, Y. M. Salih, K. H. Hama Aziz, and S. B. Shujahadeen, Electrochemistry, 88, 447 (2020).

22. K. Haerens, E. Matthijs, K. Binnemans, and B. V. der Bruggen, Green Chem., 11, 1357 (2009). 